\title{
HOT DUST ON THE OUTSKIRTS OF THE BLR IN FAIRALL 9
}

\author{
J. Clavel \& W. Wamsteker \\ ESA IUE Observatory \\ Madrid, Spain
}

\author{
I. Glass \\ SAAO \\ South-Africa
}

\section{Observations and data analysis}

Since 1978, the Seyfert I galaxy F9 $(z=0.0461)$ has been observed at 54 epochs in the far ultraviolet and optical range with the IUE (Boggess et al 1978), and 27 times in the near IR from SAAO. The IUE provided spectrophotometric low resolution (900 km.s $\left.{ }^{-1}\right)$ datá over the 1200-3200 $\AA$ range as well as simultaneous broad band optical photometry at an effective wavelength of $5200 \AA$. SAAO data consist of photometric measurements in the $\mathrm{J}, \mathrm{H}, \mathrm{K}$ and $\mathrm{L}$ bands. From the IUE data, we obtained the light-curves of the continuum at $1328 \AA$ and $1826 \AA$ (rest wavelengths), as well as those of the Ly $\alpha 1216$, CIV1550 and MgII2800 emission lines. More details can be found in Clavel, Wamsteker and Glass (1989).

\section{The ultraviolet-optical continuum}

The UV continuum varied by a factor $f=F_{\max } / F_{\min }=33 \pm 5$ at $1328 \AA$ and $1826 \AA$. The variations were very regular, with a maximum in 1978.5, a long decline with a deep minimum in mid-1984 and a continuous rise since then. Despite large variations of its intensity, the shape of the UV continuum remained constant to a high level of accuracy. This shape can be characterized by a constant spectral index $\left(F_{\nu} \propto \nu^{\alpha}\right): \alpha=-0.46 \pm 0.11$, or alternatively by a black-body temperature of $26500 \pm 900 \mathrm{~K}$, interestingly close to the mean value in the sample of AGN's studied by Edelson and Malkan (1986). The constancy of the colour temperature rules-out thermal instabilities in an accretion disc as the origin of the UV variations. On the other hand, the variations are much too rapid to be explained by viscous instabilities. The variations of the optical and $\mathbf{J}$ flux are highly correlated and simultaneous with those of the ultraviolet continuum. However, the amplitude of the variations are an order of magnitude smaller at $5200 \AA$ and $1.2 \mu$, with $f=2.86 \pm 0.27$ and $1.98 \pm 0.08$ respectively. This cannot be accounted for entirely by star light dilution, unless the stellar population is exceptionally bright and peculiar. Alternatively, a steep and non-variable IR power-law (Edelson and Malkan 1987) could account for the reduced variability at optical and near IR wavelengths.

\section{The near IR continuum}

The variations of the $\mathrm{H}, \mathrm{K}$ and $\mathrm{L}$ flux are also highly correlated with those in the ultraviolet. However, they appear to be delayed with respect to the UV variations. The lag increases with wavelength (Figure 1), from $250 \pm 100$ days at $1.65 \mu$, to $385 \pm 100$ days at $2.2 \mu$ and 
$410 \pm 100$ days at $3.5 \mu$. The amplitude of the near IR variations are the largest at $\mathrm{K}$. These results almost certainly imply that the near IR flux is dominated by thermal radiation from : dust grains heated by and at a mean distance of $400 \pm$ 100 lt-d from the UV source. Other scenarios, such as o̊ those of Puetter and Hubbard (1985) or Collin-Souffrin (1987) face serious difficulties in explaining the observations. The equilibrium temperature of the dust is $1730 \pm 230 \mathrm{~K}$, i.e. that of graphite grains at their evaporation temperature. The mass of hot dust is small (about $0.02 M_{o}$ ),

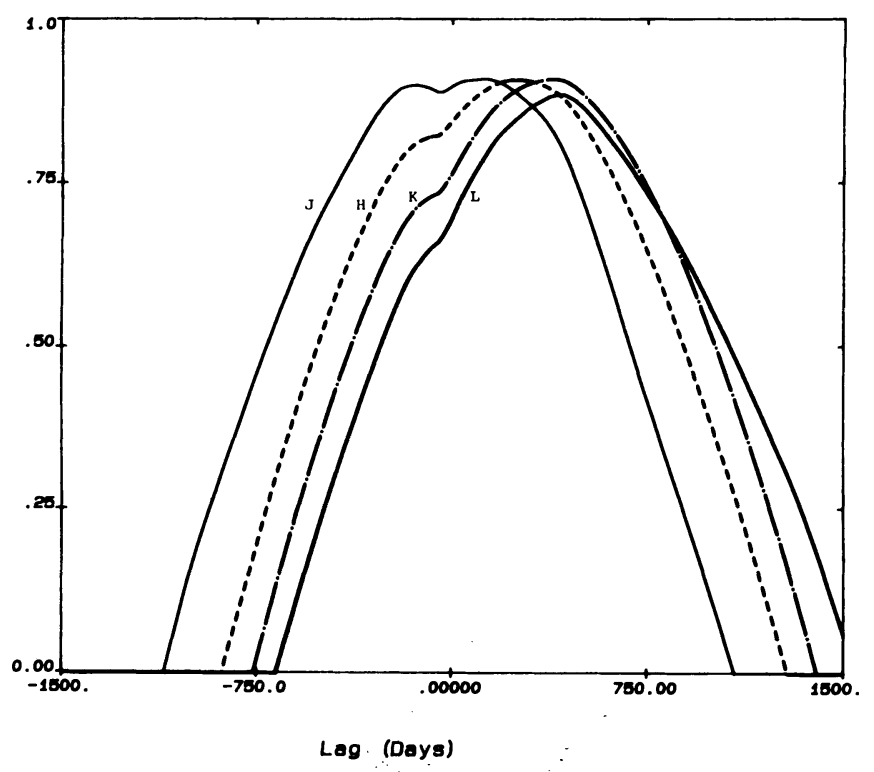

Figure 1. The Cross-Correlation functions of the $\mathrm{J}, \mathrm{H}$, $\mathrm{K}$ and $\mathrm{L}$ light-curves with that of the $\mathrm{UV}$ continuum. Note the lag increasing from $O$ at $\mathrm{J}$ to 400 days at $\mathrm{L}$.

but the far IR energy distribution of F9 suggests that a much larger reservoir of warm grains exists throughout the NLR up to distances of 300pc (Barvainis 1987).

\section{The BLR}

The variations of the Ly $\alpha 1216$, CIV1550 and MgII2800 emission line intensities are highly correlated with but lag behind those of the UV continuum, by $115 \pm 70,200 \pm 80$ and $160 \pm 90$ days respectively. This strongly suggests that the broad line gas is photoionized by and lies at a mean distance of $155 \pm 45 \mathrm{lt}$ - $d$ from the UV source. We therefore conclude (i) that the BLR gas is dust free and (ii) that the shell of hot dust lies at the periphery of the BLR, presumably at its interface with the NLR. There exist strong positive correlations between the $L y \alpha / C I V$ and $L y \alpha / M g I I$ intensity ratios on the one hand, and the UV continuum flux on the other. Photoionization models do not predict such correlations unless the shape of the ionizing spectrum varies dramatically with its intensity. This is indeed what happened: during the 1978-1984 fading of F9, the $0.5-10 \mathrm{Kev}$ flux dropped by a factor of 3.5 only (Morini et al 1986 and our EXOSAT data). In other words, the soft $X$-ray-to-UV ratio increased by an order of magnitude. The above correlations put strong constraints on the shape of the ionizing spectrum in the unobserved EUV range and imply that the flat $U V$ continuum must roll over sharply before $50 \mathrm{eV}$. 


\section{DISCUSSION}

GASKELL 1) I think it is likely that $\mathrm{Mg}$ II does come from a larger region. In our paper on Fairall 9 (Koratkar and Gaskell, Ap. J., submitted) we find a larger size for Mg II than C IV at the one or two sigma level (as was the case for NGC 4151). Indirect evidence comes from the fact that $\mathrm{Mg}$ II is usually narrower than C IV. 2) You could be finding a real lag between the optical and the UV.

CLAVEL 1) I have been using your definition of the error attached to the lag, and with that definition, the differences between the "size" of the $\mathrm{Mg} \| \lambda 2800$ and C IV $\lambda 1550$ line emitting region is not significant. That fact that $\mathrm{Mg}$ II is narrower than C IV of course fits nicely into a model where the BLR is stratified in velocity and degree of ionization, but it cannot be used as a proof that the $\mathrm{Mg}$ II gas lies further away. 2) The small shoulder at positive lag on the CCF of the optical light-curve is probably not significant. In any case, the FES bandpass is broad and includes delayed $\mathrm{H} \alpha$ and $\mathrm{H} \beta$ emission, which could be the reason for the shoulder. 